\title{
MIMO INTERACTIONS IN SAMPLED DATA SYSTEMS
}

\author{
Mario Salgado Brocal ${ }^{*, 1}$ Diego Rojas Oyarzún *,2
}

\author{
* Department of Electronic Engineering \\ Universidad Técnica Federico Santa María \\ Valparaíso, Chile
}

\begin{abstract}
In this paper, a MIMO interaction measure is used to analyze how the interaction evolves in the context of sampled data control, as a function of the sampling frequency. The basic tool is the Participation Matrix, which is a measure of the dynamic interaction in stable MIMO systems. This measure provides support for decentralized input-output pairing as well as for a richer controller architecture selection, including triangular, block diagonal and sparse structures. It is shown that the controller structure selection may be significantly dependant on the sampling period.
\end{abstract}

Keywords: multivariable control, interaction, decentralized control, discrete-time systems, sampled-data systems.

\section{INTRODUCTION.}

One of the main challenges when designing a controller for a multivariable plant is to choose a suitable controller structure. This decision requires to quantify the significance of the interactions in the plant model. In the decentralized (diagonal) architecture, this issue translates into how inputs and outputs are paired. In the general case, that quantification may lead to controllers with structures ranging from decentralized up to full MIMO.

The problem of quantifying interactions in a MIMO system has received a lot of attention over the last four decades, starting with the seminal work of E. Bristol (Bristol, 1966), who developed the idea of Relative Gain Array (RGA). The field has been enriched with additional ideas such as Niederlinski index (Niederlinski, 1971). A variation of the RGA was proposed by $\mathrm{Z}$.

\footnotetext{
1 msb@elo.utfsm.cl

2 drojaso@elo.utfsm.cl
}

Zhu (Zhu, 1996). A common feature of those indices is that they only use the system model at zero frequency. Dynamic features were introduced in the Relative Dynamic Gain Array (RDGA), (Witcher and McAvoy, 1977), (Bristol, 1978). The RDGA also provides information of how interaction varies with frequency, suggesting bandwidths for alternative pairings. That line of work was continued by Gagnon and co-workers (Gagnon et al., 1999) through the Generalized Relative Dynamic Gains (GRDG).

Recently, two gramian-based interaction measures have been proposed (Conley and Salgado, 2000), (Wittenmark and Salgado, 2002), (Salgado and Conley, 2004). In (Salgado and Conley, 2004) the Participation Matrix (PM) is proposed as a tool to quantify dynamic interactions in stable multivariable systems. It is shown that the PM can be applied, in continuous time and in discrete time frameworks, to decide on which controller structure is more appropriate. In this paper the Participation Matrix is used to explore the evolu- 
tion of the multivariable dynamic interaction as a function of the sampling frequency.

The expression system structure is used in the sequel to denote the web of paths from the (vector) input to the (vector) output, independently of the dynamical order of each scalar subsystem.

We consider a discrete-time system with $p$ inputs and $p$ outputs which can be modeled as a multivariable process with matrix transfer function given by $\mathbf{G}[z]=\left[G_{i j}[z]\right]$. The same notation is used to denote the transfer function of a sampled data system, where the underlying continuous time system is described by $\mathbf{G}(s)=\left[G_{i j}(s)\right]$. A zero-order hold is assumed to be present in the sampling process.

This paper is organized as follows: in section $\S 2$ controllability and observability gramians are revisited and the main properties of the Participation Matrix are summarized. In section $\S 3$ the $\mathrm{PM}$ is used to quantify interactions in discretetime and sampled data systems. Those results are illustrated with examples in $\S 4$. Conclusions are presented in section $\S 5$.

\section{PARTICIPATION MATRIX}

\subsection{Gramians}

Gramians, in linear control theory, are matrices which describe controllability and observability properties of a given stable linear system. They can be computed for continuous-time and discrete-time systems.

Assume that a $p \times p$ stable, discrete-time MIMO system has a state space representation given by the 4-tuple $\left(\mathbf{A} \in \mathbb{C}^{n \times n}, \mathbf{B} \in \mathbb{C}^{n \times p}, \mathbf{C} \in \mathbb{C}^{p \times n}\right.$, $\mathbf{0} \in \mathbb{R}^{p \times p}$ ), then the controllability gramian, $\mathbf{P}$, and the observability gramian, $\mathbf{Q}$, are hermitian non negative definite matrices which satisfy the Lyapunov equations (1)-(2)

$$
\begin{aligned}
& \mathbf{P}-\mathbf{A P A}^{H}-\mathbf{B} \mathbf{B}^{H}=0 \\
& \mathbf{Q}-\mathbf{A}^{H} \mathbf{Q A}-\mathbf{C}^{H} \mathbf{C}=0
\end{aligned}
$$

Useful interpretations for gramians can be derived using energy concepts (see (Glover, 1984) and (Salgado and Conley, 2004)).

Gramians quantify how hard is to control and to observe the system state, and the ranks of $\mathbf{P}$ and $\mathbf{Q}$ are the dimensions of the controllable subspace and observable subspace respectively. However, gramians depend on the state space realization. To extract valuable information, the product $\mathbf{P Q}$ is formed and its eigenvalues, $\lambda_{i}(i=1,2, \ldots, n)$, are computed. It can be proved that those eigenvalues are non-negative and that they do not depend on the particular realization, see e.g. (Glover, 1984) and (Kwakernaak and Sivan, 1972). The system Hankel singular values (HSV) are defined as

$$
\sigma_{H}^{(i)}=\sqrt{\lambda_{i}} \quad i=1,2, \ldots, n
$$

where the $\lambda_{i}^{\prime} s$ are ordered to obtain $\sigma_{H}^{(1)} \geq \sigma_{H}^{(2)} \geq$ $\ldots \geq \sigma_{H}^{(m)} \geq 0$.

It turns out that the system gramians are strongly connected to the gramians of the elementary subsystems. To define these subsystems, consider the system state space description (A, B, C, 0), where

$$
\mathbf{B}=\left[\begin{array}{lllll}
\mathbf{b}_{1} & \mathbf{b}_{2} & \ldots & \mathbf{b}_{\mathbf{p}}
\end{array}\right] ; \quad \mathbf{C}^{H}=\left[\begin{array}{llll}
\mathbf{c}_{1} & \mathbf{c}_{2} & \ldots & \mathbf{c}_{\mathbf{p}}
\end{array}\right]
$$

We can then associate with the MIMO system, a set of elementary (SISO) subsystems, each of them having a single input $u_{j}, j \in\{1,2, \ldots, p\}$, and a single output $y_{i}, i \in\{1,2, \ldots, p\}$, and a state space model given by $\left(\mathbf{A}, \mathbf{b}_{\mathbf{j}}, \mathbf{c}_{\mathbf{i}}{ }^{H}, 0\right)$ with gramians $\mathbf{P}_{\mathbf{j}}$ and $\mathbf{Q}_{\mathbf{i}}$ satisfying

$$
\begin{aligned}
& \mathbf{P}_{\mathbf{j}}-\mathbf{A P}_{\mathbf{j}} \mathbf{A}^{H}-\mathbf{b}_{\mathbf{j}} \mathbf{b}_{\mathbf{j}}{ }^{H}=\mathbf{0} \\
& \mathbf{Q}_{\mathbf{i}}-\mathbf{A}^{H} \mathbf{Q}_{\mathbf{i}} \mathbf{A}-\mathbf{c}_{\mathbf{i}} \mathbf{c}_{\mathbf{i}}{ }^{H}=\mathbf{0}
\end{aligned}
$$

A key observation is that $G_{i j}[z]$ is the transfer function of the minimal realization of the elementary subsystem with input $u_{j}$ and output $y_{i}$. Hence, the sets of HSV for the elementary subsystem $\left(\mathbf{A}, \mathbf{b}_{\mathbf{j}}, \mathbf{c}_{\mathbf{i}}{ }^{T}, 0\right)$ and for the minimal system, characterized by $G_{i j}[z]$ differ only in a subset of zero HSV.

The connection between the system gramians and those of the elementary subsystems is described by the following lemma.

Lemma 2.1. (Gramian decomposition). Let $\mathbf{P}_{\mathbf{j}}$ and $\mathbf{Q}_{\mathbf{i}}$ be the controllability and observability gramians for the elementary system $\left(\mathbf{A}, \mathbf{b}_{\mathbf{j}}, \mathbf{c}_{\mathbf{i}}{ }^{T}, 0\right)$ given by (4).

Then, the original system controllability and observability gramians $\mathbf{P}$ and $\mathbf{Q}$ are $\mathbf{P}=\sum_{j=1}^{p} \mathbf{P}_{\mathbf{j}}$, and $\mathbf{Q}=\sum_{i=1}^{p} \mathbf{Q}_{\mathbf{i}}$.

\section{Proof. See (Salgado and Conley, 2004).}

From the gramians decomposition introduced in Lemma 2.1, it can be seen that the product $\mathbf{P Q}$ for the multivariable process is given by (7)

$$
\mathbf{P Q}=\left(\sum_{j=1}^{p} \mathbf{P}_{\mathbf{j}}\right)\left(\sum_{i=1}^{p} \mathbf{Q}_{\mathbf{i}}\right)=\sum_{i=1}^{p} \sum_{j=1}^{p} \mathbf{P}_{\mathbf{j}} \mathbf{Q}_{\mathbf{i}}
$$


The maximum controllability and observability is attained when a full MIMO controller architecture is chosen, then all terms of the form $\mathbf{P}_{\mathbf{j}} \mathbf{Q}_{\mathbf{i}}$ have to be added to compute the system HSV. However if a restricted complexity controller is chosen, then only a subset of those terms is involved.

\subsection{Interaction quantification}

The above analysis requires, to have practical interest, a way to quantify and to compare.

By definition, the products $\mathbf{P Q}$ and $\mathbf{P}_{\mathbf{j}} \mathbf{Q}_{\mathbf{i}}$ have non negative eigenvalues. However, the eigenvalues of a sum of products $\mathbf{P}_{\mathbf{j}} \mathbf{Q}_{\mathbf{i}}$ is not equal, in general, to the sum of the eigenvalues of each summand. It turns out that the trace of the product $\mathbf{P}_{\mathbf{j}} \mathbf{Q}_{\mathbf{i}}$ is a convenient basis to measure the interaction and the degrees of controllability and observability of different controller structures. Recall that the trace of $\mathbf{P}_{\mathbf{j}} \mathbf{Q}_{\mathbf{i}}$ is the sum of the squared HSV for the elementary system with input $j$ and output $i$ and also recall that the HSV associated to the pair $\left(\mathbf{P}_{\mathbf{j}}, \mathbf{Q}_{\mathbf{i}}\right)$ quantify the combined abilities of input $u_{j}$ and output $y_{i}$ to control and to observe the system state.

The information encompassed in the above results can be organized in a matrix $\boldsymbol{\Phi}=\left[\phi_{i j}\right] \in \mathbb{R}^{p \times p}$, which is known as the participation matrix (PM), defined by

$$
\phi_{i j}=\frac{\operatorname{trace}\left[P_{j} Q_{i}\right]}{\operatorname{trace}[P Q]}
$$

where the trace measure has been normalized by $\operatorname{trace}(\mathbf{P Q})$. This implies that $0<\phi_{i j}<1$ and that $\sum_{i=1}^{p} \sum_{j=1}^{p} \phi_{i j}=1$.

From (8) we observe that $\phi_{i j}$ quantifies the relative dynamic relevance of the subsystem $(i, j)$. It is then crucial to understand the dependence of trace $\left[P_{j} Q_{i}\right]$ on dynamic features such as pole and zero locations, d.c. gain and delays. For sampled data systems, those features, except the d.c. gain, depend on the sampling frequency. The interplay between those dynamic features, the sampling period and trace $\left[P_{j} Q_{i}\right]$ defines the focus of this paper.

\section{PM AND DISCRETE-TIME SYSTEMS}

In the PM framework, the selection of a model with captures the most significant interactions in a MIMO system is a competitive game. Indeed, a given elementary subsystem with transfer function $G_{i j}[z]$ has to compete with other subsystems to be included in the model with the chosen structure. In this paper, we explore how the compet- itive stand of that particular subsystem changes, as the sampling frequency is modified.

For the purpose stated above, we compute and discuss the indicator $\operatorname{trace} P_{j} Q_{i}$ for certain classes of elementary subsystems.

\subsection{First order case}

Consider a first order system with transfer function given by

$$
G_{i j}[z]=K \frac{1-a}{z-a}
$$

Using (5) and (6) with $\mathbf{A}=a, \mathbf{B}=K(1-a)$ and $\mathbf{C}=1$ we obtain

$$
\operatorname{trace}\left\{P_{j} Q_{i}\right\}=K^{2} \frac{1}{(1+a)^{2}}
$$

This results says that the significance of this elementary subsystem will increase as the pole approaches -1 . This property is particularly interesting, since in the continuous-time counterpart trace $P_{j} Q_{i}$ does not depend on the location of the pole, but only on the subsystem d.c. gain. Equation (10) also verifies that the HSV is proportional to the d.c. gain $K$.

Consider a system model $G_{i j}[z]$ which arises from sampling a first order continuous-time system with pole at $s=p \in \mathbb{R}^{-}$, i.e. with time constant $\tau=|p|^{-1}$ and $a=e^{-\frac{\Delta}{\tau}}$. Therefore, the dynamic relevance of the sampled-data system is larger for those continuous-time systems with larger bandwidths. If $\Delta>>\tau$, then trace $\left\{P_{j} Q_{i}\right\}$ tends to $K^{2}$. On the contrary, if $\Delta<<\tau$, then trace $\left\{P_{j} Q_{i}\right\}$ tends to $0.25 K^{2}$, which is the corresponding value for the continuous time system (Salgado and Conley, 2004). On the other hand, if a sampled data system has only one stable pole in the interval $(-1,0)$, we then now that the underlying continuous time system has damped oscillatory modes, and that the sampling frequency has been ill-chosen.

\subsection{Second order case}

Consider a second order system with transfer function given by

$$
G_{i j}[z]=\frac{K_{1}}{z-a_{1}}+\frac{K_{2}}{z-a_{2}}
$$

where $K_{1} \in \mathbb{C}, K_{1} \in \mathbb{C}, a_{1} \in \mathbb{C}$ and $a_{2} \in \mathbb{C}$.

The model in (11) accommodates systems with and without a zero, and with real and complex poles. However we will always require that $G_{i j}[z] \in \mathbb{R}$, for all $z \in \mathbb{R}$. 


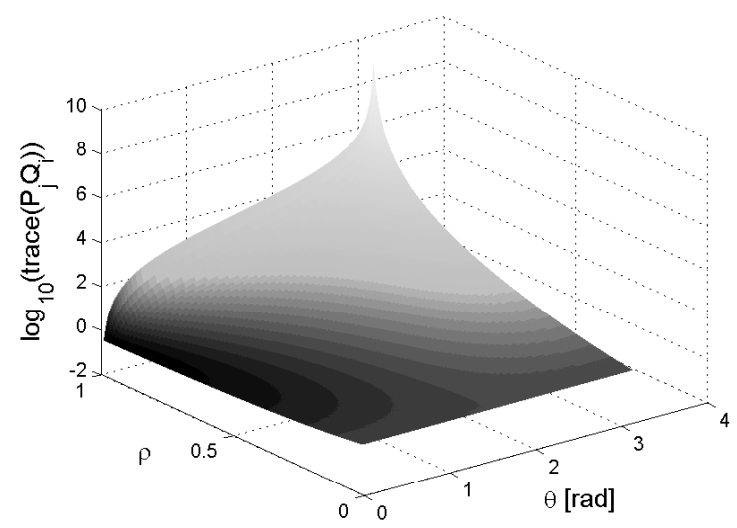

Fig. 1. System with two complex poles and no zero

Using (5) and (6) with $\mathbf{A}=\left[\begin{array}{cc}a_{1} & 0 \\ 0 & a_{2}\end{array}\right] ; \quad \mathbf{B}=$ $\left[\begin{array}{ll}K_{1} & K_{2}\end{array}\right]^{T} ; \mathbf{C}=\left[\begin{array}{ll}1 & 1\end{array}\right]$, we obtain

$\mathbf{P}_{\mathbf{j}}=\left[\begin{array}{cc}\frac{\left|K_{1}\right|^{2}}{1-\left|a_{1}\right|^{2}} & \frac{K_{1} K_{2}^{*}}{1-a_{1} a_{2}^{*}} \\ \frac{K_{1}^{*} K_{2}}{1-a_{1}^{*} a_{2}} & \frac{\left|K_{1}\right|^{2}}{1-\left|a_{1}\right|^{2}}\end{array}\right] ; \mathbf{Q}_{\mathbf{i}}=\left[\begin{array}{cc}\frac{1}{1-\left|a_{1}\right|^{2}} & \frac{1}{1-a_{1}^{*} a_{2}} \\ \frac{1}{1-a_{1} a_{2}^{*}} & \frac{1-\left|a_{1}\right|^{2}}{1-}\end{array}\right] ;$

To proceed with the analysis we will consider several cases.

3.2.1. Two complex poles, no zero In this case, we assume that the poles are conjugate, that is $a_{1}=a_{2}^{*}$ and that $K_{1}=-K_{2}$ to ensure that there is no zero. These assumptions and (12) lead to

$\operatorname{trace}\left\{\mathbf{P}_{\mathbf{j}} \mathbf{Q}_{\mathbf{i}}\right\}=\frac{2 K_{1}^{2}}{\left(1-\left|a_{1}\right|^{2}\right)^{2}}-\frac{K_{1}^{2}}{\left(1-a_{1}^{2}\right)^{2}}-\frac{K_{1}^{2}}{\left(1-a_{2}^{2}\right)^{2}}$

Also, to have a unit d.c. gain we need $K_{1}=$ $\frac{\left(1-a_{1}\right)\left(1-a_{2}\right)}{\left(a_{1}-a_{2}\right)}$. We then describe the poles as $a_{1}=$ $a_{2}^{*}=\rho e^{j \theta}$ and the trace is computed for different values of $\rho$ and $\theta$. The result is shown in Figure 1. It is there observed that trace $\left\{\mathbf{P}_{\mathbf{j}} \mathbf{Q}_{\mathbf{i}}\right\}$ increases as the

Figure 1 show that the largest value for $\operatorname{trace}\left\{\mathbf{P}_{\mathbf{j}} \mathbf{Q}_{\mathbf{i}}\right\}$ is achieved when the poles get close to $(-1,0)$.

3.2.2. Real poles, no zero In this case $a_{1} \in \mathbb{R}$, $a_{2} \in \mathbb{R}, a_{1} \neq a_{2}$. Since there is no zero, and given that $G[z]$ must be real for all real $z$, we have that $K_{1}=-K_{2}$. Then

$\operatorname{trace}\left\{\mathbf{P}_{\mathbf{j}} \mathbf{Q}_{\mathbf{i}}\right\}=\frac{K_{1}^{2}}{\left(1-a_{1}^{2}\right)^{2}}-\frac{K_{1}^{2}}{\left(1-a_{1} a_{2}\right)^{2}}+\frac{K_{1}^{2}}{\left(1-a_{2}^{2}\right)^{2}}$

To analyze the effect of the system d.c. gain we choose $K_{1}=\frac{\left(1-a_{1}\right)\left(1-a_{2}\right)}{a_{1}-a_{2}}$. The trace of $\mathbf{P}_{\mathbf{j}} \mathbf{Q}_{\mathbf{i}}$ is shown in Figure 2 , where it can be appreciated

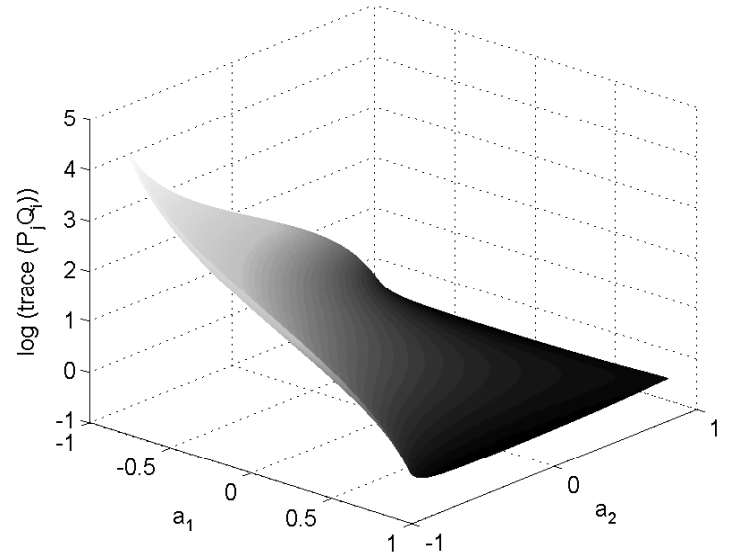

Fig. 2. System with two real poles and no zero

that $G_{i j}[z]$ has the strongest combination of controllability and observability when its two poles are close to $(-1,0)$.

3.2.3. Two real poles, one real zero In this case $a_{1} \in \mathbb{R}, a_{2} \in \mathbb{R}, a_{1} \neq a_{2}$, and we assume that $G_{i j}[z]$ has a real zero at $z=c$. If we also choose the gains $K_{1}$ and $K_{2}$ to achieve unit d.c. gain, we have that

$$
\left[\begin{array}{l}
K_{1} \\
K_{2}
\end{array}\right]=\left[\begin{array}{c}
\left(c-a_{1}\right)\left(1-a_{1}\right)\left(1-a_{2}\right) \\
\left(a_{2}-c\right)\left(1-a_{1}\right)\left(1-a_{2}\right)
\end{array}\right] \frac{1}{\left(a_{2}-a_{1}\right)(1-c)}
$$

leading to (13) with $K_{1}$ and $K_{2}$ given by (14).

In this case, trace $\left\{\mathbf{P}_{\mathbf{j}} \mathbf{Q}_{\mathbf{i}}\right\}$ achieves its maximum when the poles are close to $(-1,0)$ and the zero is close to $(1,0)$. If both poles are in the interval $[0,1)$, still that maximum is achieved for the zero close to $(1,0)$. These results are in agreement with that observed in the previous case, since the system modes are oscillatory with poor damping. Also, a zero close to the stability boundary is a strong dynamic feature in discrete and in continuous-time systems.

In sampled data control systems, the presence of a zero is sometimes originated in the sampling process. This sampling zero is usually located in the negative real axis. To explore this issue further, we consider the case of a continuous-time system with transfer function $G_{i j}(s)=\frac{1}{(s+1)(\tau s+1)}$, where $\tau$ goes from 0.1 up to 10 . We next consider a sampling process with zero-order hold and sampling period $\Delta$ going from 0.01 up to 20 . The value of $\operatorname{trace}\left\{\mathbf{P}_{\mathbf{j}} \mathbf{Q}_{\mathbf{i}}\right\}$ is shown in Figure 3 .

Figure 3 shows that if $\tau$ is fixed, then the higher combined controllability and observability is achieved for the largest sampling period. This sampling period produces a sampling zero close to the origin. In general, the closer the sampling 


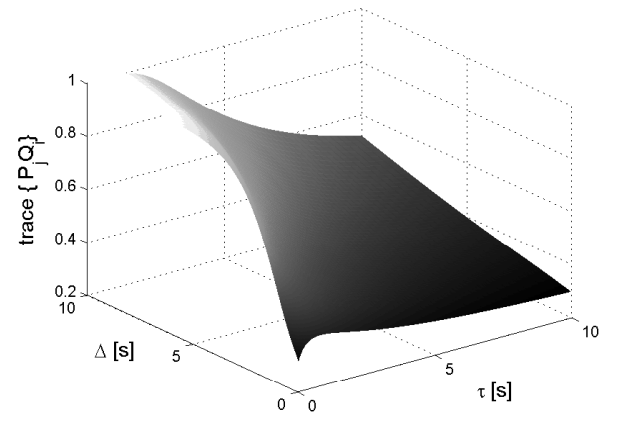

Fig. 3. $\operatorname{trace}\left\{\mathbf{P}_{\mathbf{j}} \mathbf{Q}_{\mathbf{i}}\right\}$ for a sampled-data second order system

zero is to $(1,0)$ the largest value is obtained for $\operatorname{trace}\left\{\mathbf{P}_{\mathbf{j}} \mathbf{Q}_{\mathbf{i}}\right\}$.

Two main results can be drawn form the analysis above: firstly, the value of $\operatorname{trace}\left\{\mathbf{P}_{\mathbf{j}} \mathbf{Q}_{\mathbf{i}}\right\}$ varies with the sampling frequency, and secondly, trace $\left\{\mathbf{P}_{\mathbf{j}} \mathbf{Q}_{\mathbf{i}}\right\}$ grows as the sampling frequency diminishes.

These results say that interaction in a sampleddata MIMO systems may be significantly dependant on the sampling period. This means, for instance, that the input-output pairing for decentralized control might be required to be changed if the sampling frequency changes. The general effect of this change on the PM interaction measure is illustrated in the following examples.

\section{EXAMPLES}

\subsection{Effect of large bandwidth dynamics}

Consider the continuous time full MIMO system described by its matrix transfer function

$$
G_{1}(s)=\left[\begin{array}{cc}
\frac{0.375(s+2)}{(s+1)(s+1.5)} & \frac{-5}{(s+10)} \\
\frac{4}{(s+10)} & \frac{0.375(-s+4)}{(s+1)(s+3)}
\end{array}\right]
$$

The PM of $G_{1}(s)$ is given by

$$
\Phi_{1}=\left[\begin{array}{ll}
0.2345 & 0.2212 \\
0.1416 & 0.4027
\end{array}\right]
$$

Suppose a digital control system is to be implemented, so a zero order hold model for $G_{1}(s)$ is computed with sampling period $\Delta$. Some cases are shown next regarding the effect of the sampling period election.

\subsubsection{Sampling period $\Delta=10[\mathrm{~ms}]$}

Using $\Delta=10[\mathrm{~ms}]$ the zero order hold equivalent for $G_{1}(s)$ is given by

$$
G_{1}[z]=\left[\begin{array}{cc}
\frac{0.0037406(z-0.9802)}{(z-0.99)(z-0.9851)} & \frac{-0.047581}{(z-0.9048)} \\
\frac{0.038065}{(z-0.9048)} & \frac{0.0036018(-z+1.041)}{(z-0.99)(z-0.9704)}
\end{array}\right]
$$

The PM of $H_{1 a}[z]$ is given by

$$
\Phi_{1 a}=\left[\begin{array}{ll}
0.2270 & 0.2343 \\
0.1499 & 0.3887
\end{array}\right]
$$

According to the criteria discussed in (Salgado and Conley, 2004), a reasonable structure would be to make $y_{1}$ depend on both $u_{1}$ and $u_{2}$, leaving $y_{2}$ depending just on $u_{2}$, that is to select a triangular structure for the control design stage. This selection coincides with that for the continuoustime plant.

Note that this selection has a total interaction measure given by

$$
\sum \phi=\phi_{11}+\phi_{12}+\phi_{22}=0.8501
$$

\subsubsection{Sampling period $\Delta=150[\mathrm{~ms}]$}

Using $\Delta=150[\mathrm{~ms}]$ the zero order hold equivalent for $G_{1}(s)$ is given by

$H_{1 b}[z]=\left[\begin{array}{cc}\frac{0.054098(z-0.7406)}{(z-0.8607)(z-0.7985)} & \frac{-0.38843}{(z-0.2231)} \\ \frac{0.31075}{(z-0.2231)} & \frac{0.027951(-z+1.903)}{(z-0.8607)(z-0.6376)}\end{array}\right]$

The PM for $H_{1 b}[z]$ can be computed as

$$
\Phi_{1 b}=\left[\begin{array}{ll}
0.1578 & 0.3555 \\
0.2275 & 0.2592
\end{array}\right]
$$

In this case, the structure selection yields a different result from that obtained with $\Delta=10[\mathrm{~ms}]$. Although the triangular structure is still a sensible choice, a suitable structure choice would be to make $y_{2}$ to depend on both inputs while considering $y_{1}$ to depend only on $u_{2}$. The achieved interaction measure is then

$$
\sum \phi=\phi_{21}+\phi_{12}+\phi_{22}=0.8422
$$

This example shows that for a sufficiently high sampling frequency, the model structure selection may be the same to that selected for the underlying continuous-time system. However, for larger sampling periods, the faster elementary subsystems start to gain more significance, possibly changing the control structure choice. Also, in agreement with intuition and known dynamical features characteristics, the nonminimum phase subsystem loses importance as the sampling period decreases.

\subsection{3x3 system}

Consider the $3 \times 3$ full MIMO continuous time system with transfer function

$$
G_{2}(s)=\left[\begin{array}{ccc}
\frac{1.5}{s+2} & \frac{12}{s^{2}+5.6 s+16} & \frac{1}{(s+1)^{2}} \\
\frac{15}{(s+5)^{2}} & \frac{3}{s+4} & \frac{0.8(-s+1.5)}{(s+1)(s+2)} \\
\frac{7.2}{(s+2)(s+4)} & \frac{0.7}{(s+1)^{2}} & \frac{10}{s^{2}+5.6 s+16}
\end{array}\right]
$$


The PM for $G_{2}(s)$ is computed as

$$
\Phi_{2}=\left[\begin{array}{lll}
0.0705 & 0.1425 & 0.1880 \\
0.0677 & 0.0705 & 0.1231 \\
0.1466 & 0.0921 & 0.0989
\end{array}\right]
$$

Some cases are shown next regarding the effect of the sampling period selection when a digital control system is to be implemented.

\subsubsection{Sampling period $\Delta=100[\mathrm{~ms}]$}

The zero order hold equivalent for $G_{2}(s)$ with the selected sampling period is given by

$$
\left[\begin{array}{ccc}
\frac{0.136}{z-0.8187} & \frac{0.04957 z+0.0411}{z^{2}-1.45 z+0.5712} & \frac{0.004679 z+0.004377}{z^{2}-1.81 z+0.8187} \\
\frac{0.05412 z+0.03877}{z^{2}-1.213 z+0.3679} & \frac{0.2473}{z-0.6703} & \frac{-0.06345 z+0.0738}{z^{2}-1.724 z+0.7408} \\
\frac{0.02957 z+0.02421}{z^{2}-1.489 z+0.5488} & \frac{0.003275 z+0.003064}{z^{2}-1.81 z+0.8187} & \frac{0.04131 z+0.03425}{z^{2}-1.45 z+0.5712}
\end{array}\right]
$$

and its PM can be calculated as

$$
\Phi_{2 a}=\left[\begin{array}{lll}
0.0764 & 0.1446 & 0.1741 \\
0.0701 & 0.0906 & 0.1154 \\
0.1432 & 0.0853 & 0.1004
\end{array}\right]
$$

Assuming we wish to use a triangular controller structure, the best choice is given by the total interaction measure

$\sum \phi=\phi_{12}+\phi_{13}+\phi_{23}+\phi_{31}+\phi_{32}+\phi_{33}=0.7630$

\subsubsection{Sampling period $\Delta=400[\mathrm{~ms}]$}

The zero order hold equivalent for $G_{2}(s)$ with $\Delta=400[\mathrm{~ms}]$ is

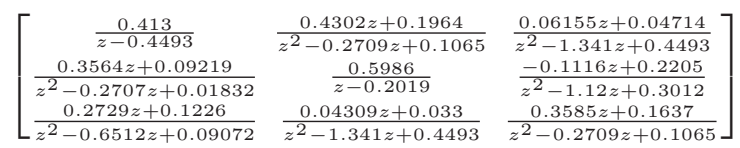

and its PM result

$$
\Phi_{2 b}=\left[\begin{array}{lll}
0.0951 & 0.1379 & 0.1501 \\
0.0726 & 0.1383 & 0.1012 \\
0.1353 & 0.0736 & 0.0958
\end{array}\right]
$$

With a triangular controller structure, the best achievable interaction measure is

$\sum \phi=\phi_{12}+\phi_{13}+\phi_{22}+\phi_{31}+\phi_{32}+\phi_{33}=0.7310$

Clearly, the controller structure decision based on the PM can be heavily dependent on the sampling time selection. In this case the nonminimum-phase zero in the subsystem $G_{23}(s)$ loses importance as the sampling period increases.

\section{CONCLUSIONS}

This paper has shown that the sampling period may substantially modify the dynamic multivariable interaction as measured by the Participation
Matrix. This is consistent with intuition since faster elementary subsystems become more relevant as the sampling frequency decreases.

\section{ACKNOWLEDGEMENTS}

The authors gratefully acknowledge the support received through grants FONDECYT-1040313 and USM-230425.

\section{REFERENCES}

Bristol, E. H. (1966). On a new measure of interaction for multivariable process control. IEEE Transactions on Automatic Control 11, 133134.

Bristol, E. H. (1978). Recent results on interaction in multivariable process control. 71st AIChE Conf.

Conley, A. and M. Salgado (2000). A grammian based interactiton measure. In: Proceedings of the 39th IEEE CDC. Vol. 5. pp. 5020-5022. Sydney - Australia.

Gagnon, Eric, André Desbiens and André Pomerleau (1999). Selection of pairing and constrained robust decentralized PI controllers. In: Proceedings of the American Control Conference.

Glover, K. (1984). All optimal hankel norm approximations of linear multivariable systems and their $l^{\infty}$-error bound. International Journal of Control 39(6), 1115-1193.

Kwakernaak, H. and R. Sivan (1972). Linear Optimal Control Systems. Wiley-Interscience. New York.

Niederlinski, A. (1971). A heuristic approach to the design of linear multivariable interacting control systems. Automatica 7, 691-701.

Salgado, Mario and Arthur Conley (2004). MIMO interaction measure and controller structure selection. International Journal of Control $77(4), 367-383$.

Witcher, M. F. and T. J. McAvoy (1977). Interacting control systems: Steady state and dynamic measure of interaction. ISA Transactions 16(3), 35-41.

Wittenmark, B. and M. Salgado (2002). Hankel norm based interaction measure for inputoutput pairing. In: IFAC 15th Triennial World Congress. Barcelona - Spain.

Zhu, Zhong-Xiang (1996). Variable pairing selection based on individual and overall interaction measures. Industrial Engineering in Chemical Research 35, 4091-4099. 